\title{
Motion Induced Artifact Mimicking Cervical Dens Fracture on the CT Scan: A Case Report
}

\author{
Yoshihisa Sugimoto, Yasuo Ito, Yasuyuki Shiozaki, Tetsuya Shimokawa, Tetsuro Mazaki \\ Department of Orthopaedic Surgery, Kobe Red Cross Hospital, Hyogo, Japan
}

\begin{abstract}
The diagnostic performance of helical computed tomography (CT) is excellent. However, some artifacts have been reported, such as motion, beam hardening and scatter artifacts. We herein report a case of motion-induced artifact mimicking cervical dens fracture. A 60-year-old man was involved in a motorcycle accident that resulted in cervical spinal cord injury and quadriplegia. Reconstructed CT images of the cervical spine showed a dens fracture. We assessed axial CT in detail, and motion artifact was detected.
\end{abstract}

Key Words: Reconstruction, Motion artifact, Mimicking, Cervical fracture

\section{Introduction}

Helical computed tomography (CT) has begun to replace plain radiography as the method of choice for cervical blunt trauma screening [1-4]. Particularly, this technology is useful in patients who have lost consciousness or sustained multiple trauma [1]. CT scanning of the cervical spine for the detection of fractures and dislocations has a sensitivity of $98 \%$ and a specificity of $100 \%$ [1]. Injuries to the craniocervical junction and lower cervical injury remain difficult to assess using plain radiographs alone.

The diagnostic performance of helical CT is excellent. However, some artifacts, such as motion, beam-hardening or scatter artifacts have been reported [5-8]. We herein report a case of motion-induced artifact mimicking cervical dens fracture.

\section{Case Report}

A 60-year-old man was involved in a motorcycle accident, resulting in cervical cord injury and quadriplegia. Upon presentation, he experienced consciousness distur- bance, multiple trauma, incomplete quadriplegia (Frankel C) and nape pain. A senior spinal surgeon was consulted and subsequently requested 16-slice helical CT and magnetic resonance imaging (MRI) scanning to assess the extent of cervical cord injury, fracture and possible dislocation. He reviewed the CT (axial view) and MRI images (Fig. 1), and diagnosed the patient with cervical spinal cord injury without fracture and dislocation. Sagittal plane CT was reconstructed subsequently, but the spinal surgeon was unable to review these images. During a conference the subsequently morning, another physician showed the sagittal CT images that were suggestive of dens fracture (Fig. 2), which were not demonstrated by MRI. A radiologist then assessed the axial $\mathrm{CT}$ in detail, and commented that motion artifacts could have contributed to images suggestive of dens fracture. The patient underwent repeat CT the subsequent day, which excluded show dens fracture (Fig. 3). This patient sustained multiple trauma and disturbance of consciousness, so he moved at first time $\mathrm{CT}$ assessment.

Received Feb 26, 2011; Revised Apr 12, 2011; Accepted May 9, 2011

Corresponding author: Yoshihisa Sugimoto, MD

Department of Orthopaedic Surgery, Kobe Red Cross Hospital,

2-5-1 Shikata-cho, Kita-ku, Okayama 700-8558, Japan

Tel: +81-86-223-9727, Fax: +81-86-235-7273, E-mail: yoshihisa64@hotmail.com 


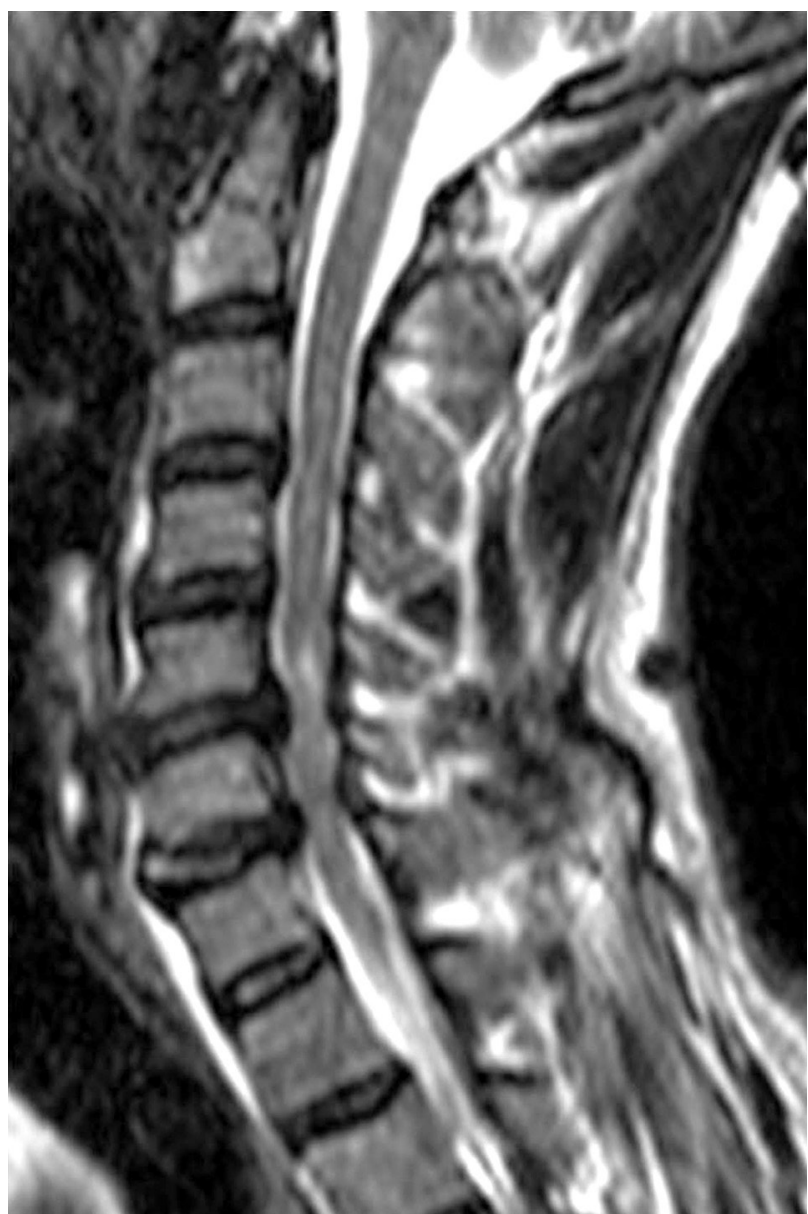

Fig. 1. Sagittal T2-weighted magnetic resonance image revealing neither fracture nor dislocation. Multi level cervical spinal canal stenoses, especially at $\mathrm{C} 5 / 6$ and $\mathrm{C} 6 / 7$, were observed.

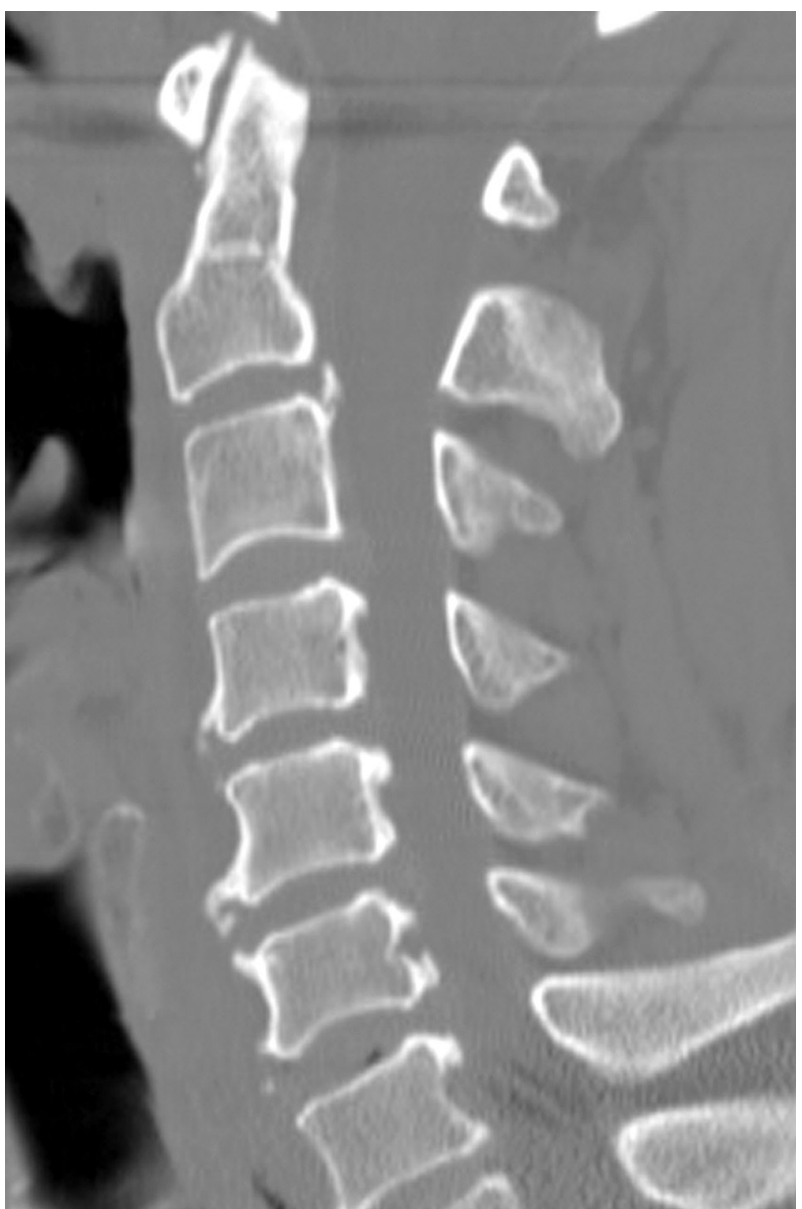

Fig. 3. Sagittal reconstruction of second computed tomography (CT) assessment. Sagittal reconstruction did not show motion-induced artifacts mimicking cervical dens fracture.

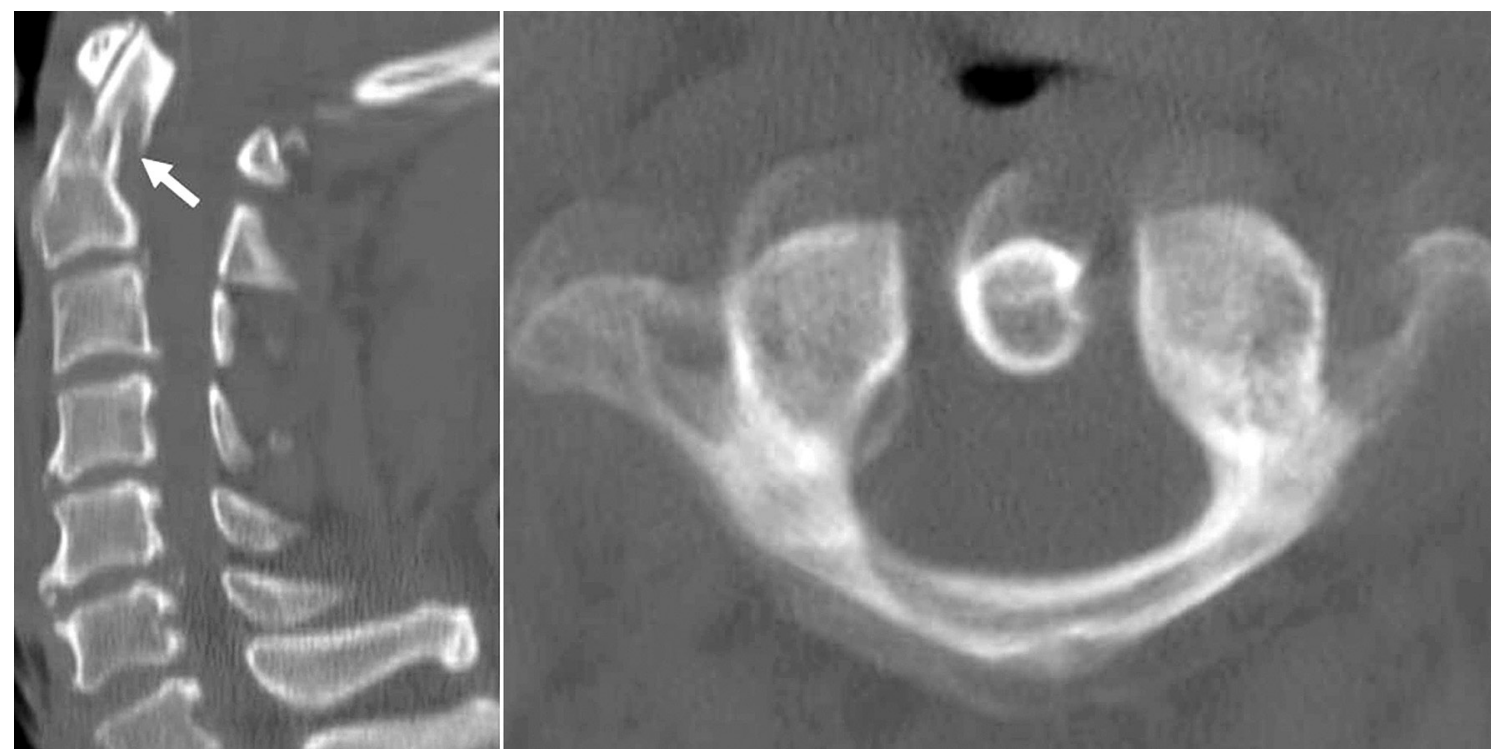

Fig. 2. Sagittal reconstruction of computed tomography (CT) images showing motion-induced artifacts mimicking cervical dens fracture (white arrow). A radiologist assessed the axial CT in detail, and motion artifact was detected. 


\section{Discussion}

Today, a wide range of traumatic and non-traumatic emergency conditions can be rapidly and accurately diagnosed by helical CT. Many traditional emergency imaging procedures have been replaced by novel helical CT techniques that can be performed quickly and with great accuracy, causing less patient discomfort and at lower costs $[1,6,9]$. The pooled sensitivity for cervical spine plain radiography is $52 \%$, and that for CT is 98\% [9] in the identification of patients with cervical spine injury. Daffner et al. [10] reviewed the CT and plain radiographic images of 245 patients, and reported that radiography detected injuries in 108 patients (44.1\%) whereas CT detected injuries in 243 patients $(99.2 \%)$ ). If a patient experiences complete or incomplete paralysis in four limbs, we always request CT and MRI assessment. Minor fractures, such as tear drop or transverse foramen fractures, can be clearly detected by CT, while cervical spinal cord injury without fracture and dislocation can be detected by MRI.

Helical CT has limitations. The first is motion artifacts [5,6]. Sciubba et al. [5] reported that CT reconstruction artifacts can mimic cervical spine subluxation. Daffner [6] showed motion artifact mimicking vertebral offset at $\mathrm{C} 3 / 4$. Voluntary or involuntary patient movements can cause such artifact mimicking cervical injury.

The second limitation is difficulty detecting fracture line parallel to the plane of the scan $[6,10]$. The third limitation is beam hardening or scatter artifacts [5]. Daffner et al. [10] reviewed the $\mathrm{CT}$ images of 245 patients with cervical injury. Two fractures not detected by CT occurred at C2: one fracture was obscured by dental artifacts and the other was in the horizontal plane of the scan [10].

In our patient, motion artifacts mimicked dens fracture, and special caution should be exercised when assessing reconstructed CT. Assessment using other imaging techniques, such as plain radiographs and MRI, is helpful in detecting motion artifacts on CT [6].

\section{REFERENCES}

1. Spiteri V, Kotnis R, Singh P, et al. Cervical dynamic screening in spinal clearance: now redundant. J Trauma 2006;61:1171-7.

2. Sekula RF Jr, Daffner RH, Quigley MR, et al. Exclusion of cervical spine instability in patients with blunt trauma with normal multidetector CT (MDCT) and radiography. Br J Neurosurg 2008;22:669-74.

3. Rana AR, Drongowski R, Breckner G, Ehrlich PF. Traumatic cervical spine injuries: characteristics of missed injuries. J Pediatr Surg 2009;44:151-5.

4. Brohi K, Healy M, Fotheringham T, et al. Helical computed tomographic scanning for the evaluation of the cervical spine in the unconscious, intubated trauma patient. J Trauma 2005;58:897-901.

5. Sciubba DM, Dorsi MJ, Kretzer R, Belzberg AJ. Computed tomography reconstruction artifact suggesting cervical spine subluxation. J Neurosurg Spine 2008;8:84-7.

6. Daffner RH. Controversies in cervical spine imaging in trauma patients. Emerg Radiol 2004;11:2-8.

7. Blumberg K. Pseudodissection of the aorta. Pediatr Radiol 2007;37:225-7.

8. Liu F, Cuevas C, Moss AA, Kolokythas O, Dubinsky TJ, Kinahan PE. Gas bubble motion artifact in MDCT. AJR Am J Roentgenol 2008;190:294-9.

9. Holmes JF, Akkinepalli R. Computed tomography versus plain radiography to screen for cervical spine injury: a meta-analysis. J Trauma 2005;58:902-5.

10. Daffner RH, Sciulli RL, Rodriguez A, Protetch J. Imaging for evaluation of suspected cervical spine trauma: a 2-year analysis. Injury 2006;37:652-8. 